\title{
Combining equilibrium, resampling, and analysts' views in portfolio optimization
}

\author{
José Luis Barros Fernandes, ${ }^{1}$ José Renato Haas Ornelas ${ }^{2}$ \\ and Oscar Augusto Martínez Cusicanqui ${ }^{3}$
}

\section{Introduction}

Portfolio optimization methodologies play a central role in strategic asset allocation (SAA), where it is desirable to have portfolios that are efficient, diversified, and stable. Since the development of the traditional mean-variance approach of Markowitz (1952), many improvements have been made to overcome problems such as lack of diversification and strong sensitivity of optimal portfolio weights to expected returns.

The Black and Litterman (1992) model (BL) is among the most used approaches. The idea behind this model is that expected returns are the result of two important sources of information: the first is market information in the form of equilibrium returns (implicit returns that clear out the outstanding market allocation), and the second is analysts' views, which tilt the market portfolio to another diversified portfolio compatible with investor beliefs. In this fashion, portfolio managers get an intuitive but formal model to generate optimal allocation.

However, while the BL model offers a very useful and intuitive approach to deal with asset allocation, the inputs considered for the calculation of equilibrium returns are subject to estimation error, and thus expected returns will also contain estimation error. Michaud (1998) proposed the use of a statistical tool known as resampling to deal with estimation error, which is an important source of lack of diversification in mean-variance portfolios. This technique considers that data come from a stochastic process instead of being a deterministic input as in Markowitz (1952).

This paper proposes the use of a portfolio optimization methodology which combines features of both the BL and resampling methodologies. This novel methodology allows the combination of equilibrium and investor's views as in $\mathrm{BL}$, and at same time deals with estimation risk as in Michaud (1998). Thus, it generates robust and diversified optimal allocations which are desirable properties for long-term investors such as central banks and sovereign wealth funds. We empirically test the new methodology using a sample of fixed income and equity indices, achieving very supportive results. We find strong evidence supporting the use of resampling techniques to improve standard models like BL and Markowitz, and this result is more pronounced for medium levels of risk. In general, our proposed methodologies, both with and without views, generated very competitive portfolios compared to the other methodologies, considering the three evaluation dimensions: financial efficiency, diversification, and allocation stability. For medium levels of risk, our methodologies are markedly better than others.

\footnotetext{
Central Bank of Brazil.

Central Bank of Brazil.

3 Central Bank of Bolivia.
}

The views expressed in this work are those of the authors and do not reflect those of the Banco Central do Brasil, Banco Central de Bolivia or its members. 
The remainder of this paper is as follows. The next section offers a brief literature review of asset allocation methodologies. The third section describes the BL resampling combined methodology. The fourth section describes the empirical study, including data and implementation and presents the results. The fifth section concludes the paper by reviewing the main results.

\section{Literature review}

The seminal work of Markowitz (1952) provided the first model for asset allocation, arguing that once expected returns and their joint variance were defined, a set of efficient portfolios could be generated and investors would choose the allocation according to their needs. Basically, the approach could be summarized as follows:

$\min \frac{1}{2} a^{T} V a$

subject to

$E\left(R_{a}\right)=a^{T} X$

where $x$ is the vector of expected excess returns, $a$ is the vector of allocations, and $v$ is the variance-covariance matrix of returns. Despite its mathematical simplicity, this model typically generates concentrated allocations which heavily depend on expected returns estimation. Resampling techniques (Michaud, 1998) were developed as a way to deal with estimation error. Markowitz recognized that resampling methods could be used to obtain better estimates for the inputs of the mean-variance optimization (Markowitz and Usmen, 2003).

Jorion (1991) used the Bayesian approach to overcome the weakness of expected returns estimated solely by sample information. He proposed an estimator obtained by "shrinking" the mean values toward a common value, chosen to be the expected return for the minimum variance portfolio. Kempf et al. (2002) pursued this Bayesian line and considered estimation risk as a second source of risk, determined by the heterogeneity of the market, which is represented by the standard deviation of the expected returns across risky assets. Both methods proved to generate better out-of-sample estimates for expected returns (as opposed to in-sample estimates), and produced more diversified portfolios.

Black and Litterman (1992) built a bridge between statistical methods and expert judgment by recognizing that the capital asset pricing model (CAPM) offers an excellent starting point for expected excess returns. Thus, combing CAPM with investors' views would produce intuitive and diversified allocations. For that, BL assumes that equilibrium returns (CAPM returns that clear out the market) are well described by the following anchor relationship:

$x \sim N(\Pi, \tau \Sigma)$

where $x$ is the observed returns vector, which is just a realization of the multivariate normal process with mean $\Pi$ (equilibrium returns), covariance matrix $\Sigma$, and an scale parameter $\tau$ which measures the degree of confidence the investor has with regard to equilibrium estimates (the nearer the parameter to zero, the higher the confidence in equilibrium estimates).

In addition to this, BL postulates that returns have another important source of information, coming from investor's views:

$X \sim N(Q, \Omega)$

where $Q$ denotes the vector of expected return views (this could be absolute or relative) and $\Omega$ is the uncertainty in those views. Since $\Omega$ is not an easy-to-obtain parameter, we employ the Idzorek (2004) approach, which measures the uncertainty through a degree of 
confidence and implicitly calculates $\Omega$. With both sources of information, the combined process is also a multivariate normal, as follows:

$$
x \sim N\left(\left[(\tau \Sigma)^{-1}+P^{T} \Omega^{-1} P\right]^{-1}\left[(\tau \Sigma)^{-1} \Pi+P^{T} \Omega Q\right] \cdot\left[(\tau \Sigma)^{-1}+P^{T} \Omega^{-1} P\right]^{-1}\right)
$$

where $P$ denotes the portfolio view matrix whose dimension is a function of the number of views (rows) and the number of assets (columns). Needless to say, since market capitalization offers a well-diversified portfolio, the optimal allocation (in general) will have this property, with tilts reflecting investors' views introduced in the model.

Finally, Michaud (1998) adapted the resampling statistic technique to mean-variance optimization, recognizing that return history is just a realization of the stochastic process behind it. Also, only if stationarity holds and in a large sample environment, the point estimates could statistically resemble the true distribution parameters. Suppose that we have a vector of expected excess return $x_{0}$ and a variance-covariance matrix denoted by $\Sigma_{0}$ (both estimated with a sample of returns of length $k$ ), assuming that returns come from a multivariate normal distribution (with parameters $\left(x_{0}, \Sigma_{0}\right)$ ), the procedure resamples $n$ times joint returns of length $\mathrm{k}$ and estimates different parameters $\left(\left\{\left(x_{1}, \Sigma_{1}\right),\left(x_{2}, \Sigma_{2}\right), \ldots,\left(x_{n}, \Sigma_{n}\right)\right\}\right)$, which allow us to obtain $n$ efficient frontiers. For a given portfolio, the resampled weights are given by the average of portfolio weights of the $n$ samples:

$$
a_{R}=\frac{1}{n} \sum_{i=1}^{n} a_{i}
$$

where $a_{R}$ is the vector of the assets' weights in the resampled portfolio, and $a_{i} s$ are the weights of each of the $n$ realizations.

Several out-of-sample evaluations have shown results in favor of resampling methodology, using different sets of data (see, for instance, Markowitz and Usmen, 2003; Pawley, 2005; Wolf, 2006; Fernandes and Ornelas, 2009). However, these evaluations cannot give definitive conclusions in favor of using resampling, given sampling limitations. Nevertheless, Fernandes and Ornelas (2009) and Kohli (2005) point out that resampled portfolios have two desirable characteristics for long-term investors. First, they usually generate portfolios that have greater diversification as more assets enter into the solution than in classical meanvariance efficient portfolios. Second, the model exhibits smoother transitions and less sudden shifts in allocations as return expectations change, meaning that the transaction costs of rebalancing the portfolio are typically lower. On the other hand, the traditional resampling methodology, considered as an ad hoc methodology, has been criticized because of its lack of a theoretical basis.

\section{Description of the BL resampling methodology}

Since, as already mentioned, the source of estimation error comes at the first part of the BL model and spreads out until the end, we combine the BL model with the resampling technique. This could be summarized as follows:

1. Estimate the BL expected return vector $x$ and the covariance matrix $\Sigma$ from historical inputs and possibly also in combination with analysts' views.

2. Resample from the results obtained in Step 1, by taking $n$ draws of length $L$ from a multivariate normal distribution with return vector $x$ and covariance matrix $\Sigma$.

3. For each draw $n$, calculate the new expected return and variance matrix. Because estimation error is present, these resampling estimates are different from the ones calculated in Step 1. 
4. For each of the $n$ sets of expected returns and covariance matrix calculated in Step 3, calculate the efficient frontier using traditional Markowitz optimization. The output of this step will be a set of $n$ efficient frontiers.

5. For each risk level, calculate the average portfolio weights across the $n$ efficient frontiers. These weights define the portfolios of the $\mathrm{BL}$ resampling frontier. The risk $x$ return profile of the BLR can be calculated using the expected return vector $x$ and the covariance matrix $\Sigma$ from Step 1.

The $\mathrm{BL}$ resampling methodology overcomes the highly criticized weakness of the traditional resampling approach as only an ad hoc methodology. This combined methodology has the theoretical background of an equilibrium model as in Black and Litterman (1992). At the same time, the use of resampling enriches the Bayesian BL approach to optimization, by recognizing estimation error.

\section{Empirical study}

\subsection{Data and implementation}

Our tests are based on monthly data of 15 indices of bonds and stocks from six developed countries. For bonds, we use six developed countries' government bond indices from BofA Merrill Lynch, and one US corporate bond index, also from BofA Merrill Lynch. For equities, we use the Thomson Datastream market indices from five countries, and three US equity market indices, divided by market capitalization: S\&P500 Composite, S\&P 400 Midcap, and S\&P600 Small Cap.

Table 1

Descriptive statistics

\begin{tabular}{c|c|c|c|c|c|c}
\hline Name & Market & $\begin{array}{c}\text { Instrument } \\
\text { Type }\end{array}$ & Mean & Std & Skewness & Kurtosis \\
\hline TBill 3M & USA & Bonds & 4.56 & 0.66 & -0.13 & -0.60 \\
US Govt & USA & Bonds & 7.14 & 4.91 & -0.05 & 0.57 \\
Canada Govt & CAN & Bonds & 9.80 & 9.10 & -0.45 & 1.87 \\
Australia Govt & AUS & Bonds & 11.34 & 12.59 & -0.69 & 1.83 \\
Japan Govt & JAP & Bonds & 8.39 & 13.01 & 0.51 & 1.63 \\
Germany Govt & GER & Bonds & 9.21 & 11.72 & 0.06 & 0.47 \\
UK Govt & UKG & Bonds & 9.62 & 11.93 & 0.14 & 0.73 \\
Germany Equity & GER & Equity & 10.32 & 21.00 & -0.53 & 1.32 \\
Australia Equity & AUS & Equity & 15.18 & 23.11 & -1.31 & 7.01 \\
Japan Equity & JAP & Equity & 6.68 & 23.16 & 0.37 & 0.58 \\
Canada Equity & CAN & Equity & 12.75 & 19.08 & -0.88 & 3.65 \\
UK Equity & UKG & Equity & 12.00 & 17.94 & -0.40 & 1.66 \\
SP500 & USA & Equity & 11.66 & 15.89 & -0.81 & 2.25 \\
SP600 SMALL & USA & Equity & 10.27 & 18.90 & -0.98 & 3.25 \\
SP400 MID & USA & Equity & 11.35 & 16.93 & -0.97 & 3.22 \\
US Corporate & USA & Corporates & 7.81 & 5.51 & -0.74 & 4.04 \\
\hline
\end{tabular}

This table presents descriptive information of each asset class considered in the analysis. The presented values are annualized and calculated over the whole sample period. 
The period of the sample is from January 1986 to December 2009, with a total of 288 months. The sample has data from market capitalization and total return index levels. All total return time series are calculated on a US-dollar basis, and we use the 3-month US T-Bill rate when calculating excess returns. We are aware that this assumption favors US assets. The US dollar is typically the numeraire considered by global investors, such as central banks, pension funds, and multinational institutions. Table 1 presents the descriptive statistics of each asset class considered.

The return time series used present the usual financial statistical characteristics, presenting a positive risk premium, negative skewness, and excess kurtosis. Figure 1 presents the risk return information for the considered asset classes. Note that, except for the Japan equity, there is an upward slope of the tendency line indicating the positive risk premium.

Figure 1

\section{Risk-return estimates of asset classes}

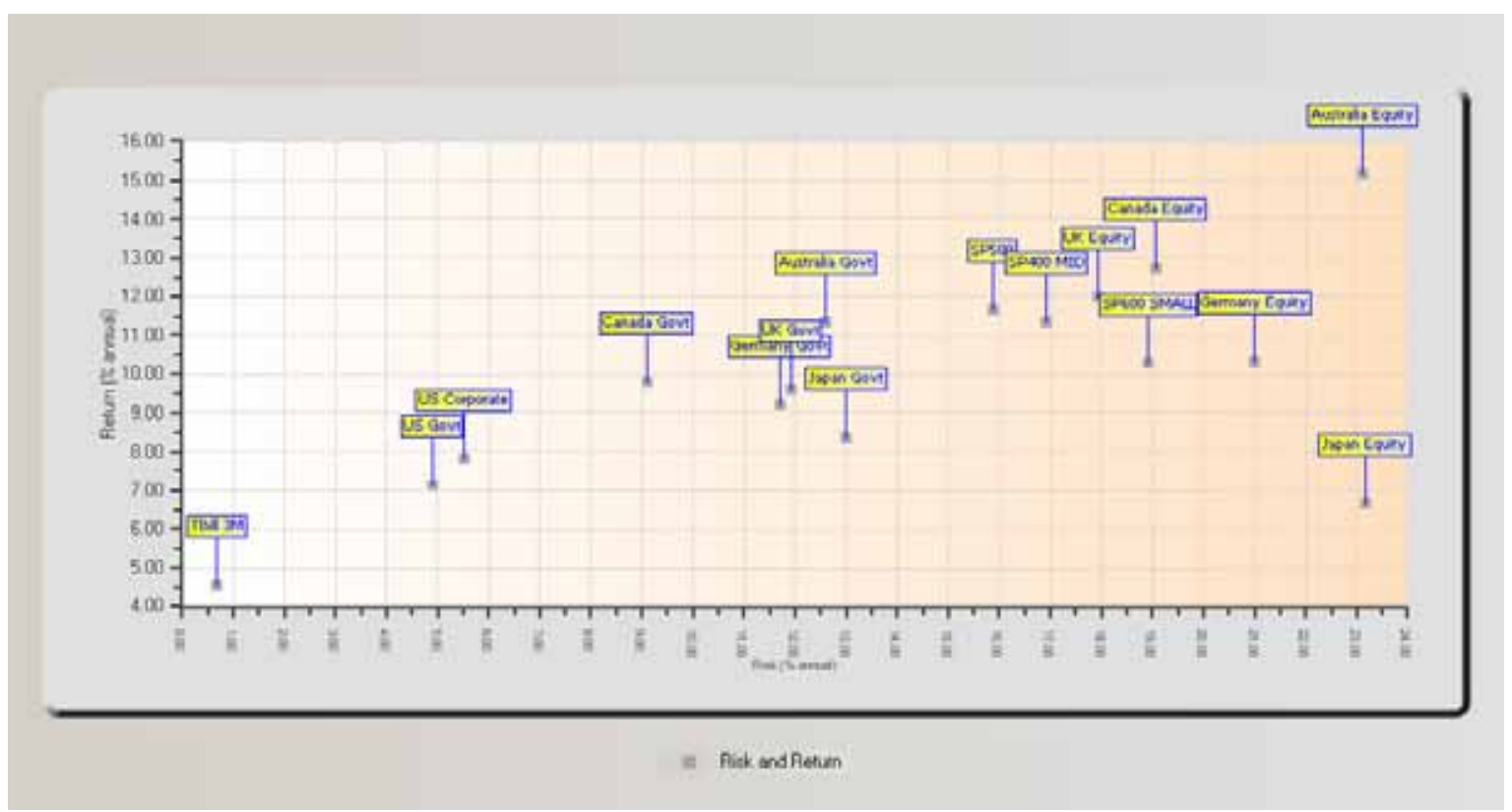

The list of methodologies that we address in this paper is shown in Table 2. The first one is the traditional Markowitz (Mark) approach (Markowitz, 1952), which uses quadratic optimization considering historical data to estimate risk and return. Resampling (Res) is the methodology proposed in Michaud (1998).

Two methodologies are based on the Black and Litterman (1992) article. The first one considers just equilibrium risk and return estimates (BL), while the second (BLView) applies the complete BL model, which combines equilibrium estimates with analysts' views. In this latter case, we consider the historical averages as the analysts' views with a confidence of $50 \%$ (we use the Idzorek (2004) framework to set the confidence level). It is important to highlight that we are not supporting any specific view methodology, but pointing out that our portfolio optimization technique may be used considering any analysts' views.

Finally, we have two methods that combine BL methodology with resampling, which were described in Section 3. The first one, BLRes, combines the BL model with the resampling methodology. The second, BLViewRes, combines the BL equilibrium model with the same analysts' views of the BLView model and also applies the resampling technique. 
Table 2

Methodologies list

\begin{tabular}{l|l}
\hline Mnemonic & Methodology \\
\hline Mark & Markowitz Portfolio Selection Model \\
Res & Portfolio Resampling Model \\
BL & BL Equilibrium Model \\
BLView & BL Equilibrium Model with Analysts' Views \\
BLRes & BL Equilibrium Model with Resampling \\
BLViewRes & BL Equilibrium Model with Analysts' Views and Resampling \\
\hline
\end{tabular}

We analyze the performance of these six optimization strategies. We vary the estimation period $(p)$ in an out-of-sample analysis. The parameters are estimated using monthly return observations of the past $p$ months. Then we define the efficient risky portfolio and hold it for the next (e) months. Then we re-estimate the parameters and adjust the portfolio weights. We evaluate the methodology's performance with a no short-selling constraint as is usual in the industry. We consider three levels of investors' risk preference (low, medium, and high) in order to infer if the results are sensitive to investors' level of risk aversion. We use an estimation period of 60 months, and evaluation periods of 6 and 12 months.

To find out which methodology outperforms the others, we take into account three evaluation dimensions - financial performance, allocation stability, and diversification - as ideally an investor would prefer stable, diversified, and financially efficient portfolios. To judge the financial performance of the strategies, we compute their empirical Sharpe ratios, and to evaluate the stability of the allocation weights generated by the methodology, we calculate the average turnover of the portfolio in the re-estimation events. To infer the diversification, we use the mean Herfindahl index, given by the sum of the squared asset allocation weights.

\subsection{Results}

Table 3 presents the results of the Sharpe ratio, Herfindahl index, and turnover for each methodology considering three levels of risk for the optimal portfolio: low (panel A), medium (panel B), and high (panel C). The estimation period ( $p$ ) considered was 60 months and the evaluation period (e) was 6 months. So, the optimizer considers the previous 5 years of data to estimate the frontier portfolios and holds the allocation for the next 6 months, for which the returns are calculated. After that, the frontier is re-estimated again considering the previous 5 years of data, and so on until the end of the sample period.

Comparing first the Markowitz (Mark) results against resampling (Res), we can see that for the three levels of risk, the Res portfolio outperformed the Mark one in all three evaluation dimensions considered. The Res portfolio turned out to be more financially efficient, diversified, and stable. This is a well-known result in the literature when we compare the traditional Markowitz approach with resampling (Markowitz and Usmen, 2003). As resampling techniques typically generate more diversified portfolios, they tend to reduce turnover and increase financial out-of-sample efficiency.

When we compare the $\mathrm{BL}$ equilibrium with the $\mathrm{BL}$ equilibrium with resampling (BLRes) results, we find out more supportive numbers related to the use of resampling. For every level of risk, the Sharpe ratio, the Herfindahl index, and the turnover are better for the BLRes.

Finally, in the results for the BL with analysts' views (BLView) and the resampled BL with analysts' views (BLViewRes) methodologies, the previous findings remain, with the BLViewRes outperforming in every dimension the BLView. 
As a general first conclusion, we found strong evidence supporting the use of resampling techniques to improve standard models, and this result is more pronounced for medium levels of risk. The reason for this might be related to the fact that for intermediate levels of risk in the frontier, we will typically find more substitute asset classes (asset classes with similar risk-return characteristics). For low and high levels of risk in the frontier, we usually find few eligible asset classes and optimal portfolios tend to be more concentrated.

Another interesting result is that our proposed resampling BL methodologies (BLRes and BLViewRes) generated very competitive portfolios when compared to the other methodologies, considering the three evaluation dimensions and the three levels of risk. Specifically for the medium level of risk, our two proposed methodologies outperform all the others in terms of Sharpe ratio and Herfindahl index. For turnover, the equilibrium BL model is the only one that outperforms our proposed methodologies.

For the high-risk portfolios (panel $\mathrm{C}$ of Table 3), the low financial performance of the methodologies based on equilibrium (BL and BLRes) is notable. It may be caused by the outlier position of the Japan equity portfolio in Figure 1, since the equilibrium approach would increase the expected returns of this asset because of its high risk. Thus, Japan equities would enter into the optimization with a higher expected return, but realized returns would still be lower. In order to check whether this low performance is related to the presence of the Japan equity in the sample, we ran the optimization exercise without Japan equities. Results for the high-risk portfolios were considerably different, and now favor BL and BLRes against Mark and Res. This is evidence that these equilibrium methodologies are very sensitive to outliers' assets in terms of risk and return.

Table 3

Results $p=60$ and $e=6$

Panel A: $60-6$ (Low)

\begin{tabular}{l|c|c|c|c|c|c}
\hline & Mark & Res & BL & BLRes & BLView & BLViewRes \\
\hline Sharpe Ratio & 0.539 & 0.579 & 0.562 & 0.600 & 0.526 & 0.586 \\
Herfindahl Index & 0.446 & 0.403 & 0.377 & 0.354 & 0.337 & 0.335 \\
Turnover & 0.308 & 0.254 & 0.242 & 0.213 & 0.281 & 0.211 \\
\hline
\end{tabular}

Panel B: $60-6$ (Medium)

\begin{tabular}{l|c|c|c|c|c|c}
\hline & Mark & Res & BL & BLRes & BLView & BLViewRes \\
\hline Sharpe Ratio & 0.367 & 0.423 & 0.402 & 0.518 & 0.388 & 0.512 \\
Herfindahl Index & 0.296 & 0.200 & 0.207 & 0.102 & 0.209 & 0.120 \\
Turnover & 0.577 & 0.375 & 0.191 & 0.208 & 0.494 & 0.259 \\
\hline
\end{tabular}

Panel C: $60-6$ (High)

\begin{tabular}{l|c|c|c|c|c|c}
\hline & Mark & Res & BL & BLRes & BLView & BLViewRes \\
\hline Sharpe Ratio & 0.346 & 0.384 & 0.174 & 0.292 & 0.318 & 0.367 \\
Herfindahl Index & 0.344 & 0.179 & 0.339 & 0.113 & 0.330 & 0.126 \\
Turnover & 0.715 & 0.459 & 0.221 & 0.249 & 0.596 & 0.326 \\
\hline
\end{tabular}

This table presents the results of the Sharpe ratio, Herfindahl index, and turnover for each methodology considering three levels of risk for the optimal portfolio: low (panel A), medium (panel B), and high (panel C). The estimation period $(p)$ considered was 60 months and the evaluation period $(e)$ was 6 months. 
To evaluate the robustness of our findings so far, Table 4 presents the results with an evaluation period of 12 months, ie the optimizer considers the previous 5 years of data to estimate the frontier portfolios and holds the allocation for the next year, for which the returns are calculated. After that, the frontier is re-estimated again considering the previous 5 years of data, and so on until the end of the sample period.

The results are pretty much in line with the ones presented in Table 3 , with resampling techniques typically improving results of the standard approaches. Again, this result is more pronounced for intermediate levels of risk. Our proposed methodologies (BLRes and BLViewRes) are still very competitive compared to the other methodologies, considering the three evaluation dimensions and the three levels of risk.

In general, Sharpe ratios are smaller when compared with the results in Table 3 . This is due to the fact that as we increase the evaluation period, the out-of-sample financial efficiency tends to diminish. In terms of diversification, the numbers are similar, so it seems that the methodologies are not affected in terms of diversification by the increase of the evaluation period. However, the turnover increased reasonably, indicating that in order to reduce transaction costs, the portfolios should be re-estimated more often.

Table 4

Results $p=60$ and $e=12$

Panel A: 60 - 12 (Low)

\begin{tabular}{l|c|c|c|c|c|c}
\hline & Mark & Res & BL & BLRes & BLView & BLViewRes \\
\hline Sharpe Ratio & 0.439 & 0.480 & 0.493 & 0.534 & 0.413 & 0.504 \\
Herfindahl Index & 0.435 & 0.390 & 0.373 & 0.337 & 0.333 & 0.320 \\
Turnover & 0.485 & 0.428 & 0.456 & 0.384 & 0.439 & 0.363 \\
\hline
\end{tabular}

Panel B: 60 - 12 (Medium)

\begin{tabular}{l|c|c|c|c|c|c}
\hline & Mark & Res & BL & BLRes & BLView & BLViewRes \\
\hline Sharpe Ratio & 0.271 & 0.351 & 0.356 & 0.429 & 0.269 & 0.428 \\
Herfindahl Index & 0.297 & 0.202 & 0.228 & 0.101 & 0.201 & 0.114 \\
Turnover & 0.853 & 0.589 & 0.376 & 0.293 & 0.727 & 0.373 \\
\hline
\end{tabular}

Panel C: 60 - 12 (High)

\begin{tabular}{l|c|c|c|c|c|c}
\hline & Mark & Res & BL & BLRes & BLView & BLViewRes \\
\hline Sharpe Ratio & 0.270 & 0.337 & 0.110 & 0.272 & 0.216 & 0.336 \\
Herfindahl Index & 0.347 & 0.188 & 0.372 & 0.118 & 0.328 & 0.128 \\
Turnover & 0.999 & 0.670 & 0.458 & 0.379 & 0.781 & 0.456 \\
\hline
\end{tabular}

This table presents the results of the Sharpe ratio, Herfindahl index, and turnover for each methodology considering three levels of risk for the optimal portfolio: low (panel A), medium (panel B), and high (panel C). The estimation period $(p)$ considered was 60 months and the evaluation period (e) was 12 months. 


\section{Conclusion}

This paper deals with a well-documented issue in mean-variance optimization, related to the fact that this methodology typically leads to unintuitive portfolios with extreme positions in asset classes. We proposed the use of an optimization approach that takes advantage of both $\mathrm{BL}$ and resampling techniques to incorporate the main positive aspects of both previous powerful techniques. It is a stochastic general equilibrium model, which can be used as a tool for both passive and active strategies. The main idea is to estimate the efficient frontier using the $\mathrm{BL}$ model but consider this frontier as just an input to the resampling method.

We empirically test our methodology using a comprehensive sample of bond and stock indices. Compared to traditional portfolio optimization methodologies, we have reached very supportive results. We found strong evidence supporting the use of resampling techniques to improve standard methodologies, and this result is more pronounced for medium levels of risk. The reason for this might be related to the fact that for intermediate levels of risk in the frontier, we will typically find more substitute asset classes (asset classes with similar riskreturn characteristics). For low and high levels of risk in the frontier, we usually find few eligible asset classes and optimal portfolios tend to be more concentrated.

Generally speaking, our proposed methodologies, both with and without views, generated very competitive portfolios compared to the other methodologies, considering the three evaluation dimensions: financial efficiency, diversification, and allocation stability. For medium levels of risk, our methodologies are markedly better than others.

It is important to highlight that a recommendation of a specific methodology for deriving analysts' views is out of the scope of the present study. The view considered in this article is just a naive example to show that the proposed methodology may be adapted to the analysts' views. We argue that the proposal of views methodologies is still an open avenue for future research in portfolio management.

\section{References}

Black, F. and Litterman, R. (1992) "Global portfolio optimization", Financial Analysts Journal, Vol. 48, No. 5, pp. 28-43.

Fernandes, J., and J. Ornelas. (2009) "Minimizing operational risk in portfolio allocation decisions". Journal of Risk Management in Financial Institutions, Vol. 2, N 4:438-450.

Jorion, P. (1991) "Bayesian and CAPM estimators of the means: Implications for portfolio selection". Journal of Banking and Finance, $N^{\circ} 15: 717-727$.

Idzorek, T. (2004) "A Step-By-Step Guide to the Black-Litterman Model: Incorporating user specified confidence levels", Zephyr Associates.

Kempf, A., Kreuzberg, K. and C. Memmel. (2002) "How to incorporate estimation risk into Markowitz optimization". Operations Research Proceedings 2001, Springer:175-192.

Kohli, Jasraj (2005) "An Empirical Analysis of Resampled Efficiency", Thesis submitted to the Faculty of the Worcester Polytechnic Institute.

Litterman, R. (2003) Modern Investment Management: An Equilibrium Approach. New York: John Wiley and Sons.

Markowitz, H. (1952) "Portfolio Selection". Journal of Finance, Vol. 7, N 1:77-91.

Markowitz, H. and Usmen, N. (2003) "Resampled Frontiers versus Diffuse Bayes: An Experiment", Journal of Investment Management, vol. 1, no. 4 (Fourth Quarter), pp. 9-25.

Michaud, R. (1998) "Efficient Asset Management", Harvard Business School Press, Boston, MA. 
Pawley, M. (2005) "Resampled mean-variance optimization and the dynamic nature of markets", paper presented at the Biennial Conference of the Economic Society of South Africa, Durban, 7-9th September.

Wolf, M. (2006) "Resampling vs shrinkage for benchmarked managers", Institute for Empirical Research in Economies, University of Zurich, Working Paper Series, No. 263. 